\title{
Research Paper \\ The Relationship Between Spiritual Well-being and Anxiety of Aged People Admitted in Coronary Care Units
}

\author{
Elham Hedayati ${ }^{1},{ }^{*}$ Maryam Hazrati ${ }^{1,2}$, Marziye Momen Nasab ${ }^{3}$, Hamid Shokoohi ${ }^{4}$, Fateme Afkari ${ }^{3}$
}

1. Department of Gerontology Nursing, Hazrate Zainab School of Nursing, Larestan University of Medical Sciences, Larestan, Iran. 2. Department of Nursing, School of Nursing and Midwifery, Shiraz University of Medical Sciences, Shiraz, Iran.

3. Department of Biostatics, School of Medicine, Shiraz University of Medical Sciences, Shiraz, Iran.

4. Department of Emergency Medicine, School of Medicine and Health Sciences, George Washington University, Washington, USA

Citation: Hedayati E, Hazrati M, Momen Nasab M, Shokoohi H, Afkari F. [The Relationship Between Spiritual Well-being and Anxiety of Aged People Admitted in Coronary Care Units (Persian)]. Iranian Journal of Ageing. 2016; 11(3):432-439. http://dx.doi.org/10.21859/sija-1103432

doi : http://dx.doi.org/10.21859/sija-1103432

Received: 15 Feb. 2016

Accepted: 26 Jun. 2016
Key words:

Anxiety, Spiritual health, Aged people

\section{A B STRACT}

Objectives Spiritual distress and anxiety are the most common problems of the elderly patients admitted in coronary care units. The elder patients are more vulnerable due to the weakening of adaptation mechanisms. Given that earlier studies on spiritual health revealed that it was related to the physical and mental health, but the relationship between spiritual health and state-trait anxiety was not clear yet. Hence, this study aimed to investigate the relationship between spiritual health and state-trait anxiety among the elderly patients admitted in coronary care units.

Methods \& Materials This study was a correlational cross-sectional study. Sixty-six elderly cardiac patients hospitalized in coronary care unit were selected by convenience sampling method. Data were collected by using Spielberger's State-Trait Anxiety Inventory, and Palutzian and Ellison Spiritual Well-being questionnaires. Analysis of data including descriptive statistics, Pearson, ANOVA t-test, and correlation coefficient was carried out using SPSS version 21. P level of less than 00.05 was considered statistically significant.

Results Most participants (69.7\%) aged between 60 to 70 years, $59.1 \%$ were female and $63.6 \%$ were married. The majority of them (57.6\%) were illiterate. The mean score of spiritual well-being was 79.55 . The mean of trait anxiety was in the mid-range of $45.18 \pm 9.21$, and the mean and standard deviation of anxiety state was in the high range of $58.44 \pm 29$.9. The results revealed a significant negative relationship between spiritual religious health, and state-trait anxiety $(P<0.05)$. The highest correlation was found between spiritual health and trait anxiety $(P<0.003)$.

Conclusion Because of the significant relationship between spiritual health and anxiety, identifying and addressing the elderly patients' spiritual needs can lead to reducing the patients' anxiety.

\section{* Corresponding Author:}

Maryam Hazrati, PhD

Address: Department of Nursing, School of Nursing and Midwifery, Shiraz University of Medical Sciences, Shiraz, Iran.

Tel: +98 (917) 3161002

E-mail: hazratim@sums.ac.ir 


\title{
ارتباط سلامت معنوى و اضطراب در سالمندان بسترى در بخش مراقبتهاى ويرٔه قلبى
}

\author{
الهام هدايتى'، "مريم حضرتى "، مرضيه مؤمننسب"، حميد شكوهى"، فاطمه افكارى"

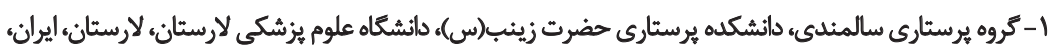

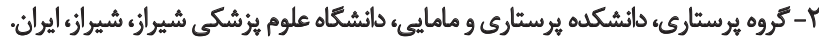

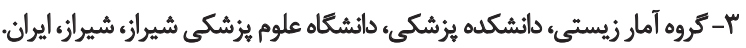

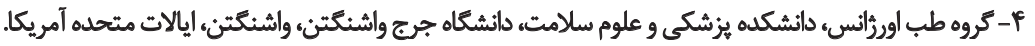

\begin{abstract}
حكSد

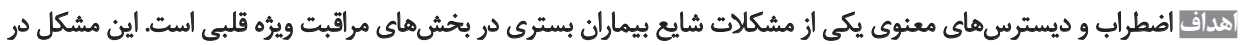

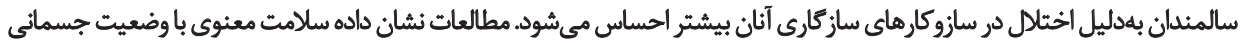

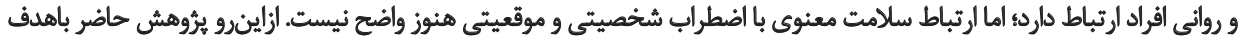

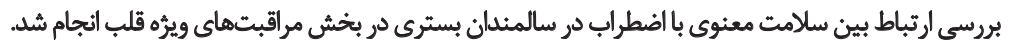

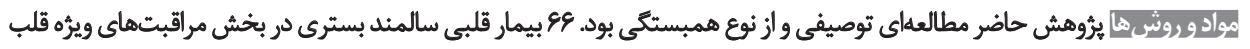

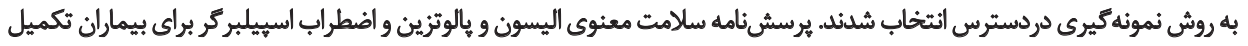

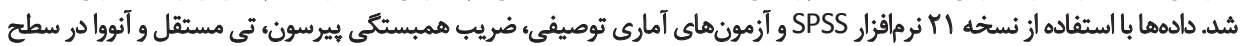

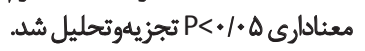

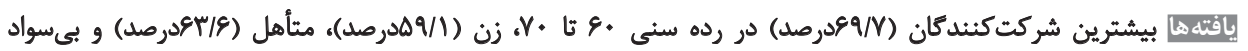

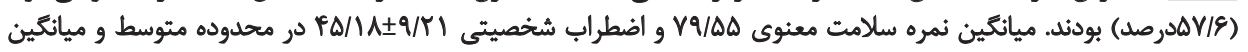

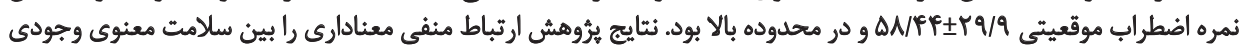

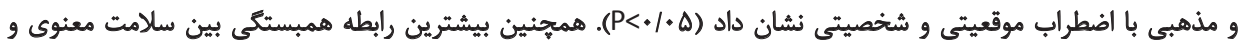

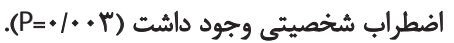

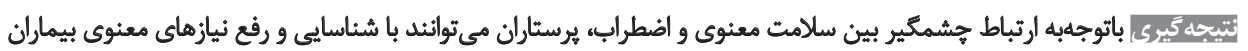

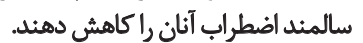

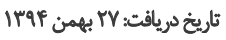

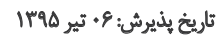

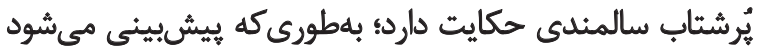

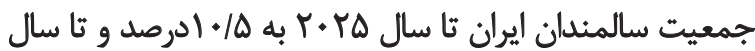

dolêم

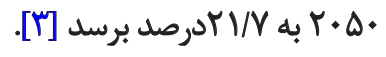

تمام انسانها طى فرايند زيستى، با فرايند اجتنابنايذيرى افزايش جمعيت سالمندى با افزايش شيوع بيمارىهاى مزمن،

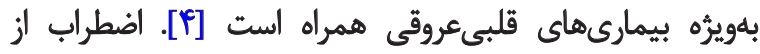

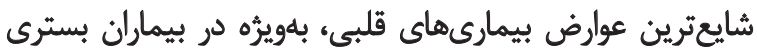

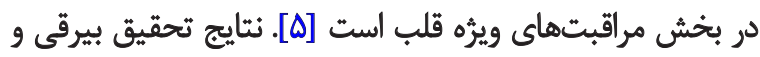

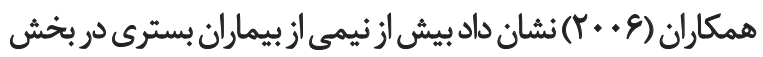

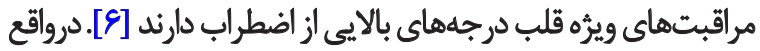

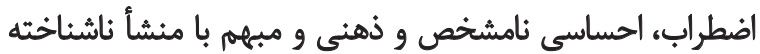

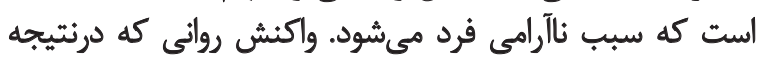

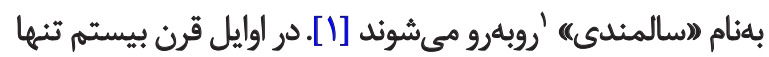

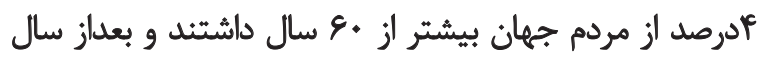

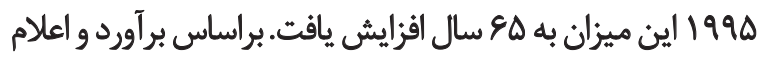

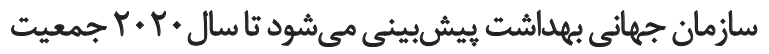

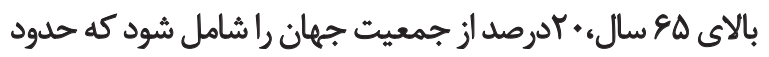

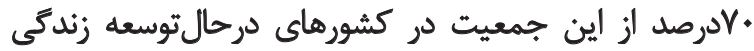

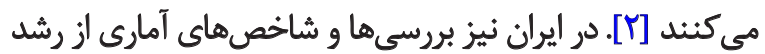

1. Aging

: نويسئده مسئول:

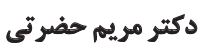

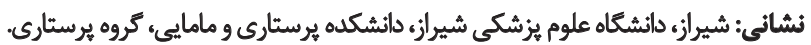

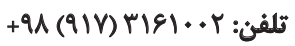
hazratim@sums.ac.ir يست الكترونيكي 
و بيمارىهاى شايع سالمندى ازجمله بيمارى هاى مختلف قلبى و

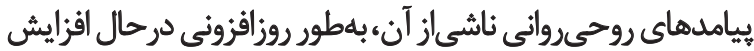

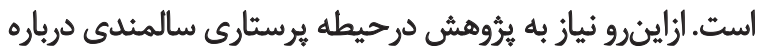

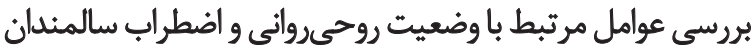

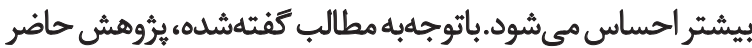

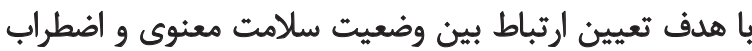

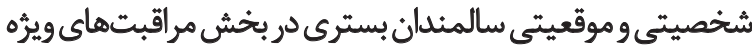

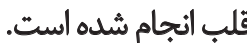

\section{روش مطالعه}

براى جمع آورى اطلاعات از سه ابزار استفاده شد:

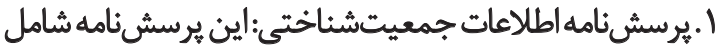
سن، جنس، وضعيت تأهل، تحصيلات و شغل است؛

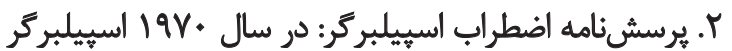

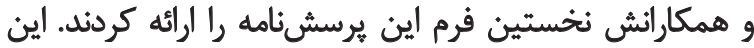

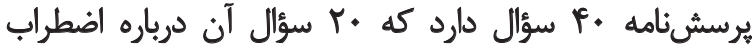

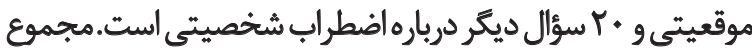

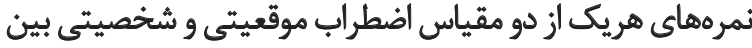

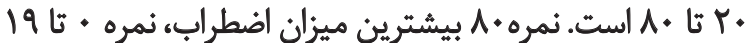

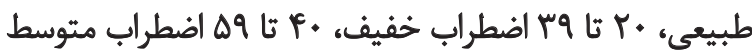

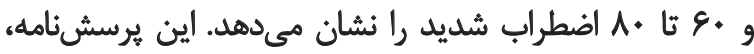

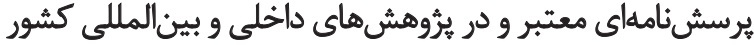

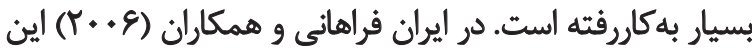

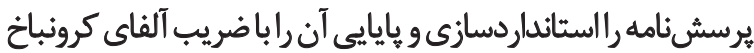

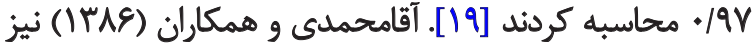

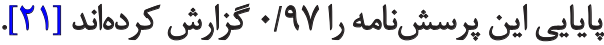

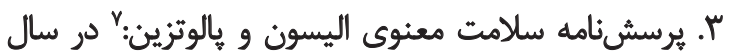

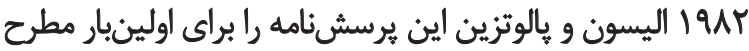

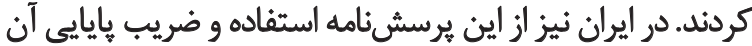

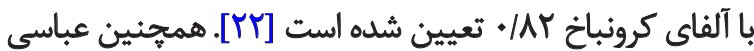

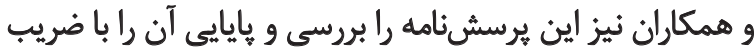

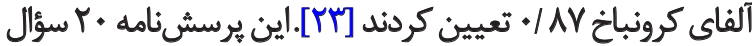

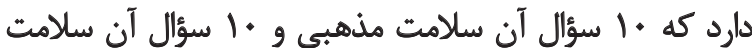

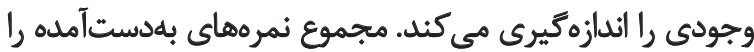

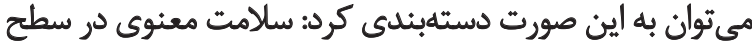

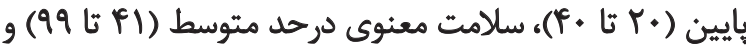

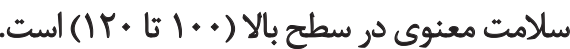

يثروهش حاضر مطالعهاى توصيفى و از نوع همبستكى است.

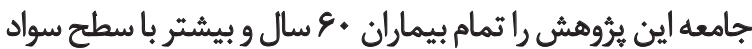

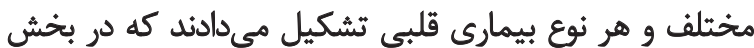

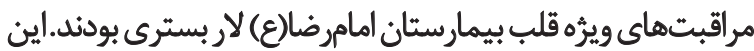

7. Palutzian and Ellison
اضطراب ايجاد مي شود، از فردى به فرد ديكر مسرى است [V] الين

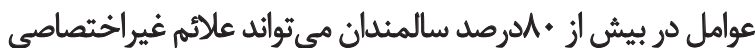

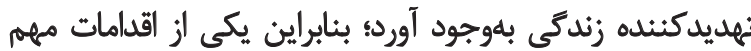

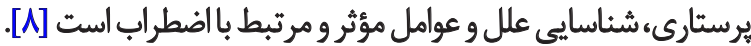
يكى از ابعاد مدنظر بسيارى از صاحبنظران براي كاهش

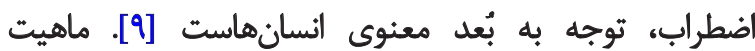

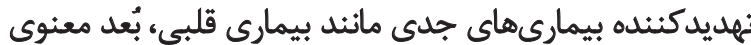

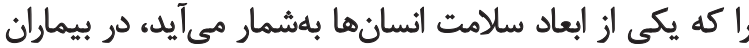

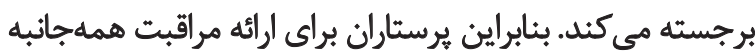

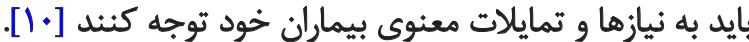

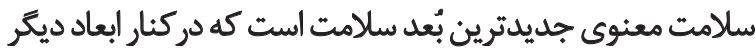

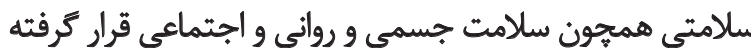

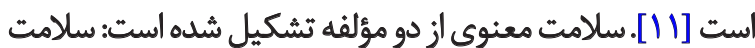

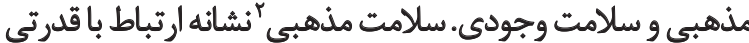

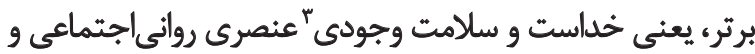

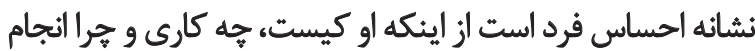

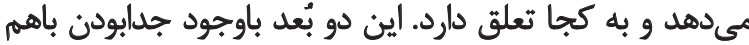

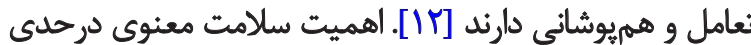

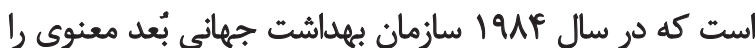

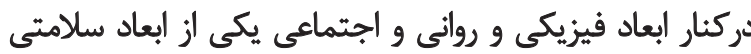

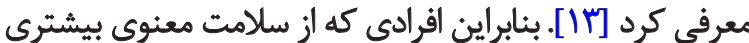

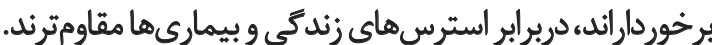
درباره ارتباط معنويت با وضعيت جسمى و روانى بيماران

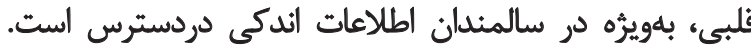

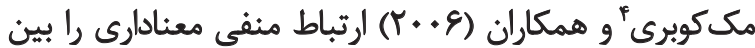

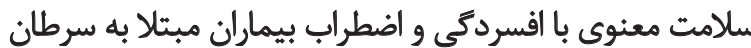

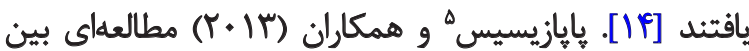

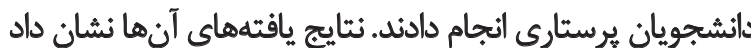

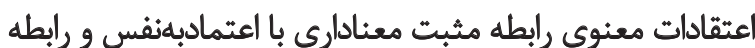

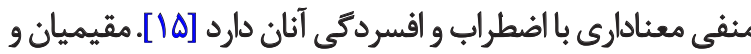

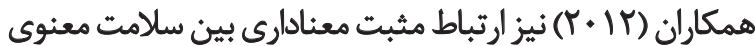

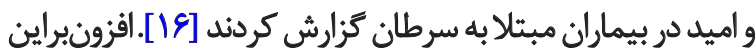

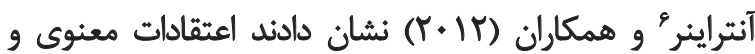

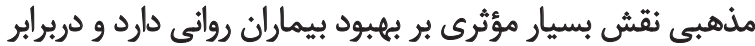

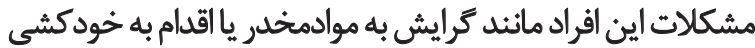
نقش حفاظتى ايفامى كند [ [IV] مطالعات نشان داد معنويت نقش مؤثرى بر كاهش اضطراب و

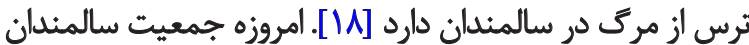

2. Religious well-being

3. Existential well-being

4. McCoubrie

5. Papazisis

6. Unterrainer 
بين افراد شركت كنثده TNA Tدرصد سطح اضطراب شخصيتى

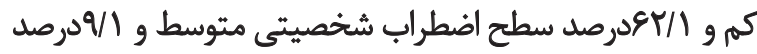

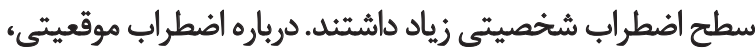

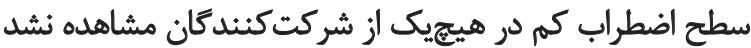

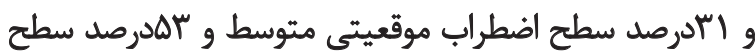

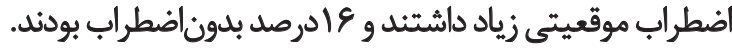
ميانكين نمره سلامت معنوى افراد VY/DQ و ميانكين نمره

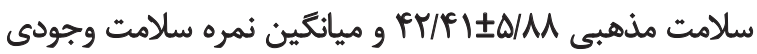
آنان

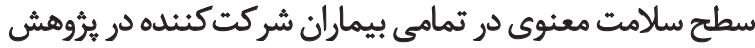

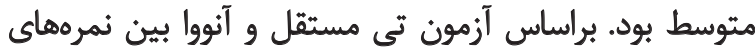

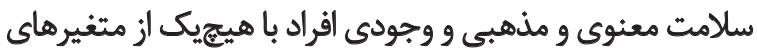

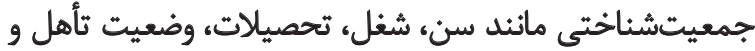

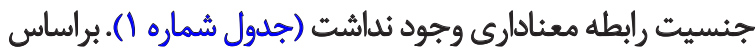

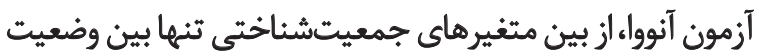

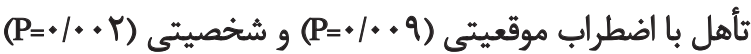

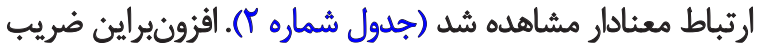

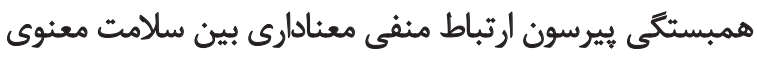

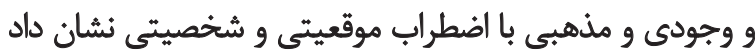

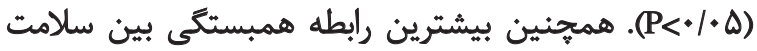

معنوى و اضطراب شخصيتى وجود داشت (جدول شماره بن) بحث

هدف از انجام اين ثروهش تعيين ميانگين و محدوده سلامت

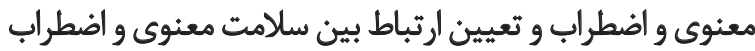

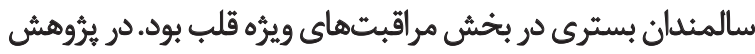

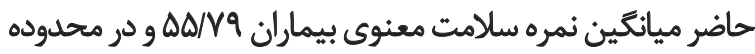

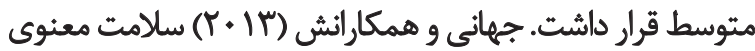

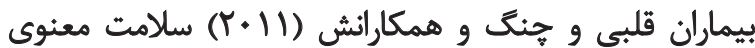

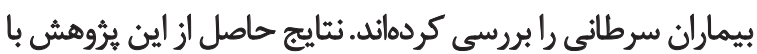

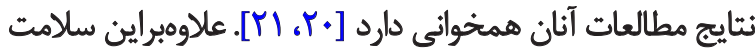

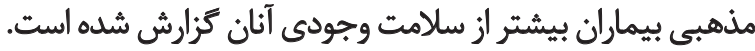

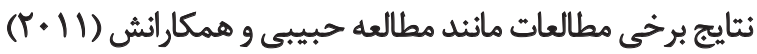

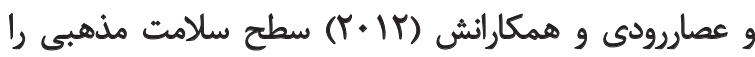

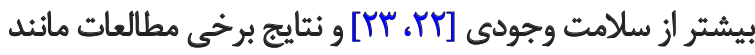

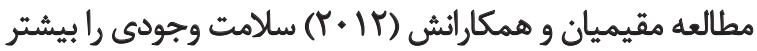

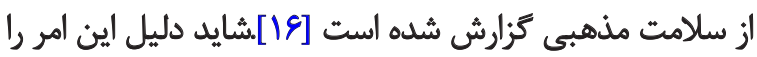

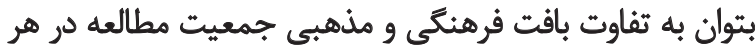

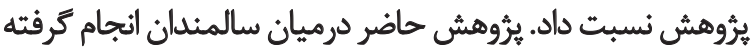

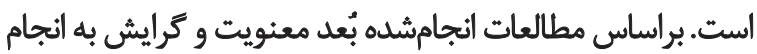

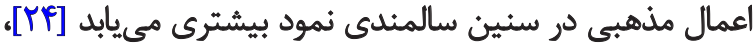

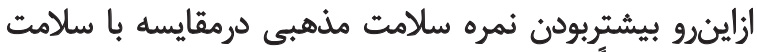
وجودى كاملاً توجيهيذير نمرد است.

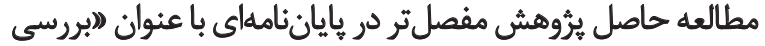

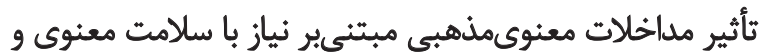

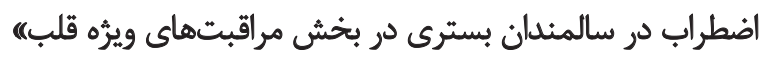

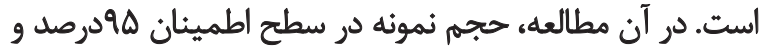

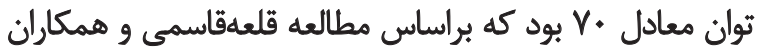

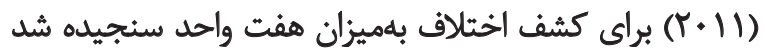

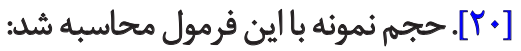

$$
\begin{gathered}
n=\frac{\left(z_{1-a / 2}+z_{1-\beta}\right)^{2}\left(\delta^{2}{ }_{1}+\delta^{2}{ }_{2}\right)}{d^{2}} \\
\delta_{1}^{2}=\delta_{2}^{2}=\frac{R}{6}=\frac{80-20}{6}=10
\end{gathered}
$$

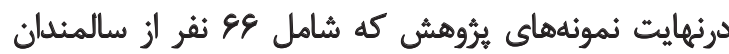

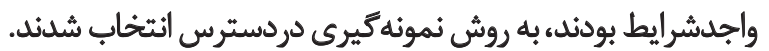

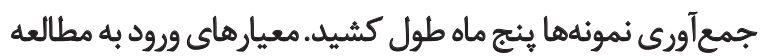

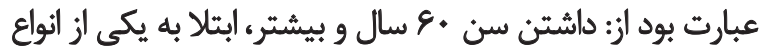

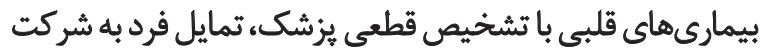

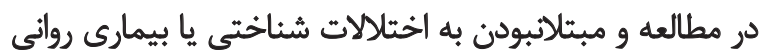

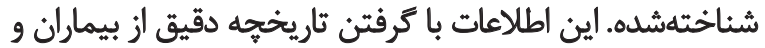
بررسى يرونده يزشكى آنان بهدست آمد.

معيارهاي خروجازمطالعهنيز شامل اين معيارهابود:انجام مداخلهها

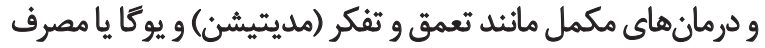

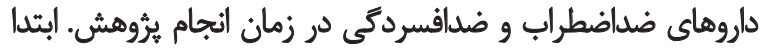

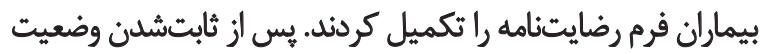

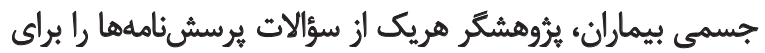

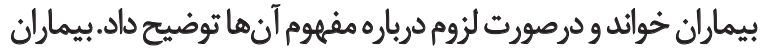

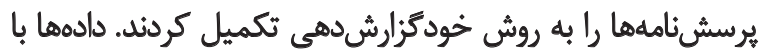

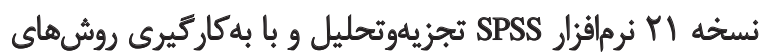

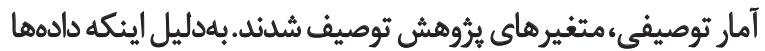

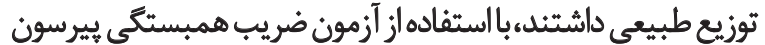

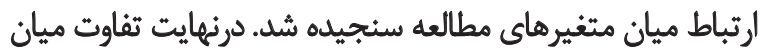

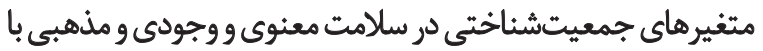

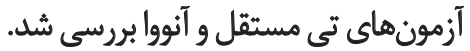

ياقتهها

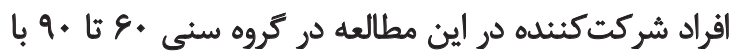
ميانكين سنى

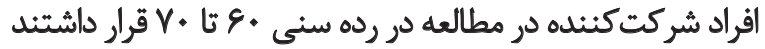

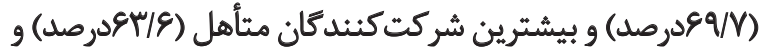

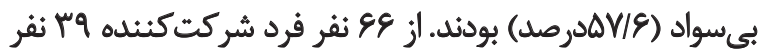

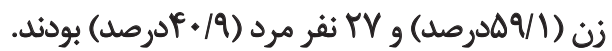

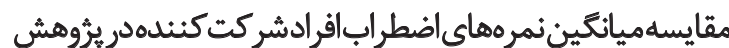

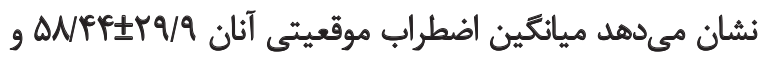

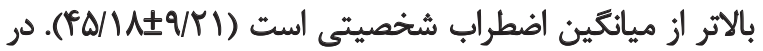


جدول ا. ارتباط بين متغيرهاي دموكّرافيك با سلامت معنوى، مذهبي و وجودى.

\begin{tabular}{|c|c|c|c|c|c|c|c|}
\hline \multicolumn{6}{|c|}{ مثثغيرها } & \multirow{2}{*}{\multicolumn{2}{|c|}{ متغير هاي جمعيتشناختى }} \\
\hline \multicolumn{2}{|r|}{ سلامت مذهبي } & \multicolumn{2}{|r|}{ سلامت وجودى } & \multicolumn{2}{|r|}{ سلامت معنوى } & & \\
\hline $\mathrm{p}$ & ميائكين و التحر اففعيار & p & ميانكين و انحرافمعيار & $\mathrm{p}$ & ميانكين و انحرافمعيار & & \\
\hline \multirow{2}{*}{ - /er } & $F T / \Delta \pm A F / M$ & \multirow{2}{*}{.10} & $r \Delta / M \pm V / N$ & \multirow{2}{*}{. Ne } & $\vee q / . V \pm 11 / T q$ & زن & \multirow{2}{*}{ جنسيت } \\
\hline & $P V / e \pm W / 19$ & & $V / M \pm V / M q$ & & $r q / A v \pm 1 \cdot \pi$ & هرد & \\
\hline \multirow{3}{*}{.$/ \Delta$} & $P V / E \pm N / M$ & \multirow{3}{*}{$\cdot / \Delta$} & TV/TAIE/AT & \multirow{3}{*}{$\cdot$ /NE } & $v q / \cdot \Delta \pm 1 \cdot / M$ & $r \cdot 58$. & \multirow{3}{*}{ سن } \\
\hline & $r T / \varepsilon \pm r \Delta / \cdot 1$ & & $\mathrm{Mf} / \Delta \mathrm{AL} \pm \mathrm{g} / \mathrm{qV}$ & & $A \cdot / A r \pm 11 / \Delta A$ & $a \cdot b n$ & \\
\hline & $r A / r \pm r \Delta / A 1$ & & $r \Delta / A V \pm I I / A \varphi$ & & $\Lambda \cdot / r \Delta \pm 11 / N \Delta$ & $9+511$ & \\
\hline \multirow{4}{*}{. $/ \Delta T^{\prime}$} & $\varphi g / r \pm \Delta / r \Lambda$ & \multirow{4}{*}{$\cdot 19$} & $r q \pm 1 . / . r$ & \multirow{4}{*}{.1 .9} & $\varepsilon \Delta \pm 1 \cdot / r T$ & مجرد & \multirow{4}{*}{ توأهل } \\
\hline & $F T / \Delta \pm+M / M F$ & & $r E / T \Delta \pm E / T A$ & & $A T / T \pm N / Y A$ & مثأهل & \\
\hline & $F / V \pm F / N E$ & & $r e / r \pm N / R$ & & $A Y \pm I r / Q \Lambda$ & مطلقه & \\
\hline & FT/VIES/FA & & $r g / \Lambda \pm q /+f$ & & $\Delta 1 / \Delta \Delta \pm 1+/ \Lambda$ & ييوه & \\
\hline \multirow{4}{*}{.1 .8} & $P T / \Delta \pm N / M q$ & \multirow{4}{*}{+19} & $r g / \Delta \Delta \pm V / \Delta \Lambda$ & \multirow{4}{*}{ ת ז//. } & $A r / r q \pm Q / r r$ & بـىسواد & \multirow{4}{*}{ نحصيلات } \\
\hline & $P r / \Delta \pm 94 / / 1$ & & $r \Delta / M \pm V / N C$ & & $N E / Q Y \pm 1 \cdot / M$ & تحصيلات إبتدايي & \\
\hline & $P r / \varphi \pm P Q / I P$ & & $r N I I \pm / \Delta P$ & & A) $/ \Delta \Delta \pm \sqrt{ } / N^{\circ}$ & دييلم & \\
\hline & $r V / \varepsilon \pm \Delta \Delta / \Psi \varepsilon$ & & 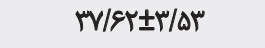 & & $V \otimes / \cdots \pm V / \cdot V$ & تحصيلات بالاتر & \\
\hline \multirow{4}{*}{$\cdot / r$} & $\mathrm{~W} / \Lambda \pm \Delta / \mu \mathrm{T}$ & \multirow{4}{*}{.$/ \pi r$} & $M \pm V / T D$ & & $n e / \Delta \pm 11 / N \wedge$ & بازئشسته & \multirow{4}{*}{ أشتثغيتل } \\
\hline & $\Gamma \Psi / \Delta \pm \Delta / \Delta \Delta$ & & rNAV $\pm \& / R T$ &.$/ 4 q$ & $A Y / / E \pm 1 \cdot / T \Delta$ & آزاد & \\
\hline & $\mu r / q \pm r g / T \Delta$ & & $r \Delta / r^{r} \pm V / r r$ & & $V Q / \Delta H \pm 11 / T \Delta$ & خانهدار & \\
\hline & $P r / P \pm \cdot / 1 q$ & & $M T / Q I \pm N E T$ & & VEl. $\pm N / Y Y$ & بيكار & \\
\hline
\end{tabular}

政

عوامل تأثيركذار بر اضطراب در بيماران قلبى است [YTV] برسلا و

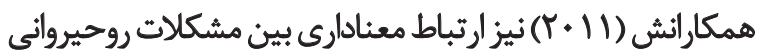

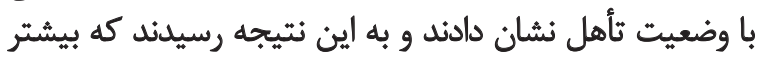

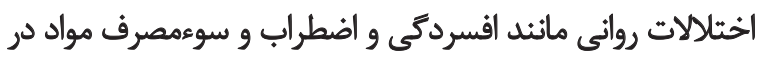

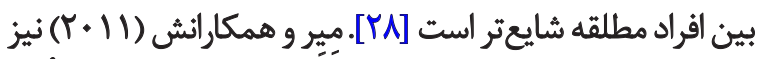

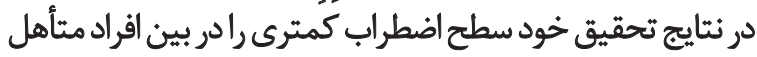

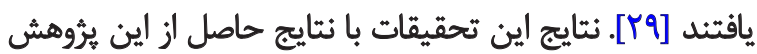

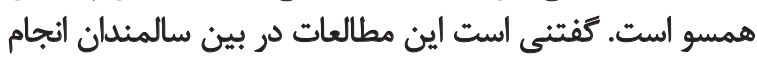

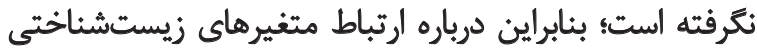

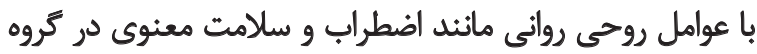
سالمندان به تحقيقات بيشترى نياز است.

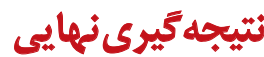

يثوهش حاضر نشان داد بين سلامت معنوى و مذهبى و

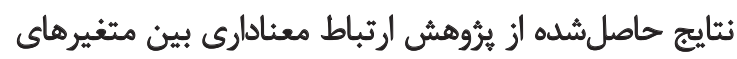

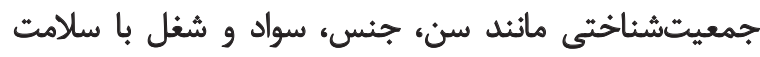

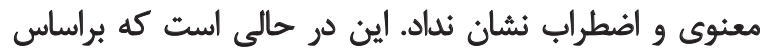

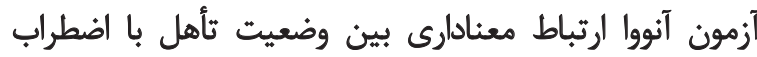

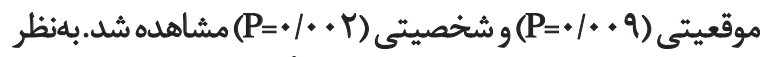

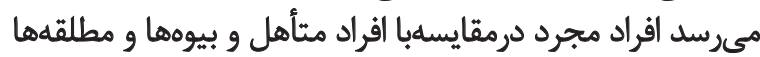

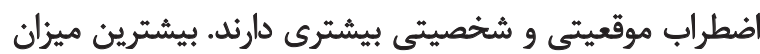

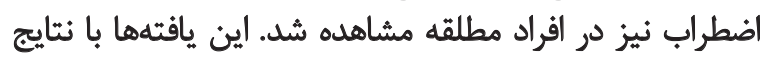

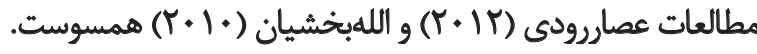

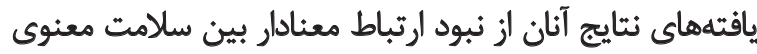

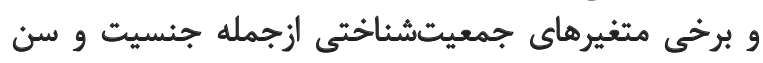

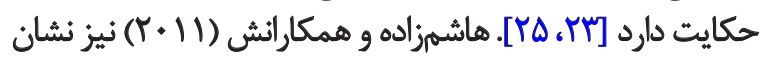

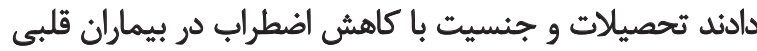

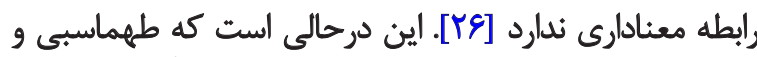

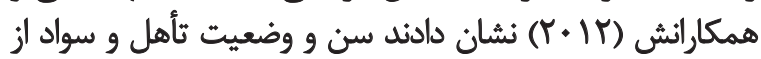


جدول r. ارتباط بين متغيرهاى جمعيتشناختى با اضطراب شخصيتى و موقعيتى.

\begin{tabular}{|c|c|c|c|c|c|}
\hline \multicolumn{4}{|c|}{ مثغيرها } & \multirow{2}{*}{\multicolumn{2}{|c|}{ متغير هاى جمعيتشناختى }} \\
\hline \multicolumn{2}{|c|}{ اضطراب موقعيتى } & \multicolumn{2}{|c|}{ اضطراب شُخصيتى } & & \\
\hline$P$ & هيانكين و أتحرافمعيار & $p$ & ميانكين و أتحر|فمعيار & & \\
\hline \multirow{3}{*}{.19} & $\Delta \Delta / 9 Y \pm I 1 / 1$ & \multirow{3}{*}{.$|g|$} & $\mu F / \Delta \Delta \pm N \Lambda \Delta$ & زن & \multirow{3}{*}{ جنسيت } \\
\hline & & & & & \\
\hline & $8 \cdot / V \pm V / R$ & & $P \Delta / 81 \pm q / \Delta)^{2}$ & مرد & \\
\hline \multirow{3}{*}{.$N$} & $\Delta V / A= \pm N Q$. & \multirow{3}{*}{$.10 A$} & Pe/AYIQ/Pr & $r+b \varepsilon$. & \multirow{3}{*}{ سن سن } \\
\hline & $\Delta \vee / 9 \pm \pm 11 / 9 \varphi$ & & PV/QAEQ/EV & A. $\mid \nabla n$ & \\
\hline & $\triangle Q / A V \pm N T \Delta$ & & Pr/grtV/D. & $9 \cdot(\forall \wedge)$ & \\
\hline \multirow{5}{*}{$.1 . .9^{*}$} & $\Delta+1+ + \pm V \mid+V$ & & $r+N \Delta \pm r / Q$ & مجرد & \multirow{5}{*}{ وضعيت تأهل } \\
\hline & $\Delta q / N \pm N+1$ & & $p+/ r \mid \pm q / * r$ & مثتأهل & \\
\hline & & $.1 . . r$ & & & \\
\hline & $g \& / F \pm Y / V A$ & & $\Delta Q / \Lambda \cdot \pm \Delta / Q \Psi$ & مطلقةه & \\
\hline & $\Delta F / F+ \pm 11 / F q$ & & $P E / T \cdot \pm V / \Delta V$ & بيوه & \\
\hline \multirow{5}{*}{.18} & QNPTII./MT & & PINIINVE & بي سواد & \multirow{5}{*}{ سطح تحصيلات } \\
\hline & QNIIINTE & & E\&/1VIII/A9 & تحصيلات إبتدايي & \\
\hline & & $+/ 9 \Delta$ & & & \\
\hline & AV/TYIV/IT & & $P \Delta / \Delta \Delta \pm V / \Delta \Delta$ & دييلم & \\
\hline & $\varepsilon \vee \pm \Delta / 8 \Delta$ & & $p 4 / . + \pm * 1 .$. & تحصيلات بالاتر & \\
\hline \multirow{5}{*}{.$/ R Y$} & gr/Ta土)./Ar & & $P V / D . \pm F M T$ & مازئشسئ & \multirow{5}{*}{ وضعيت أشتشال } \\
\hline & $\Delta Q / V+ \pm \& / A T^{H}$ & & $P \& / 18 \pm q / 9 q$ & آزاد & \\
\hline & & - ATr & & & \\
\hline & $\left.\Delta \Delta / F_{\Delta \pm}\right) \backslash / K T$ & & $r F / \Delta V \pm q /+r$ & خانهدار & \\
\hline & G./MTIV/MI & & $P T / N \Delta \pm Q M$ & بيكار & \\
\hline
\end{tabular}

L

جدول ب.ارتباط ميان متغيرهاي يروّهش.

\begin{tabular}{|c|c|c|c|c|}
\hline سلامت مذهبى & سلامت وجودى & سلامت معنوى & اضطراب شخصيتى & متغيرها \\
\hline$r=-\cdot / M r r$ & $r=-\cdot / M r V$ & $r=-\cdot / r \mid f$ & $r=* / M T r$ & \multirow{2}{*}{ اضطراب موقعيتي } \\
\hline $\mathrm{P}=+1+\mathrm{F}^{\mathrm{F}}$ & $\mathrm{P}=+1++\varphi$ & $\mathrm{P}=+1+1$ & $\mathrm{P}=+1++1$ & \\
\hline $\mathrm{r}-\cdot /$ TAF & $r=-\cdot / r \Delta$ & $r=-\cdot / r q$ & & \multirow{2}{*}{ اضطراب شخصصيتى } \\
\hline $\mathrm{P}=* 1+r$ & $\mathrm{P}=* / \cdot r$ & $P=\cdot / \cdot+r$ & & \\
\hline
\end{tabular}

乩

و كيفيت زندكى و افسردكي انجام شده است. در اين مطالعات

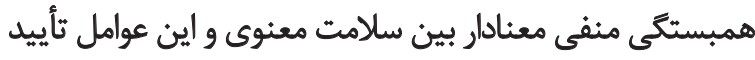

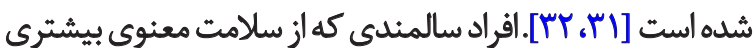

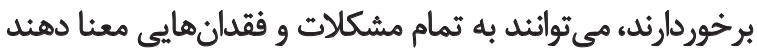

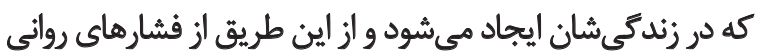

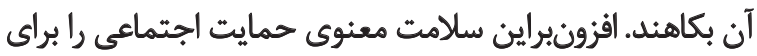

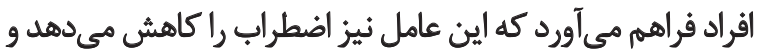

وجودى با اضطراب موقعيتى و شخصيتى ارتباط معنادار منفى إنى

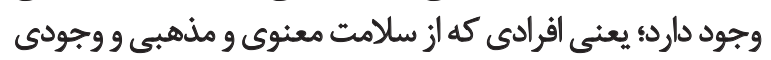

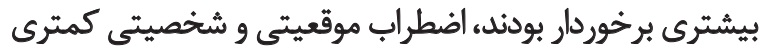

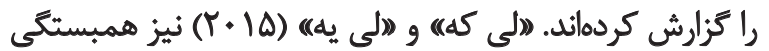

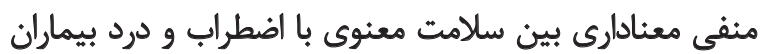

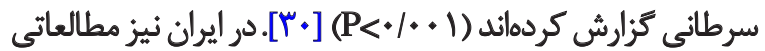

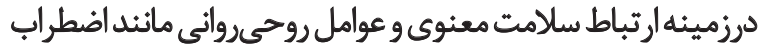




\section{References}

[1] Firooz Moghaddam S, Borgali A, Sohrabi F. [The efficiency of happiness training to increase the hope in elderly people (Persian)]. Iranian Journal of Ageing. 2014; 8(4):66-71.

[2] Gol Karami S, Mobaraki H, Kamali M, Farhoodi F. [The impact of the empowerment program on depression in the elderly day centers Sages rehabilitation city of Khorramabad (Persian)]. Journal of Modern Rehabilitation. 2013; 6(4):65-71

[3] Salehi L, Salaki S, Alizade L. [Health-related Quality of life among elderly member of elderly centers in Tehran (Persian)]. Epidemiology. 2013; 8(1):14-20.

[4] Blanton RM, Takimoto E, Aronovitz M, Thoonen R, Kass DA, Karas RH, et al. Mutation of the protein kinase i alpha leucine zipper domain produces hypertension and progressive left ventricular hypertrophy: a novel mouse model of age-dependent hypertensive heart disease. The Journals of Gerontology Series A: Biological Sciences \& Medical Sciences. 2013; 68(11):1351-355. doi: 10.1093/gerona/glt042

[5] Paudel R, Panta OB, Paudel B, Paudel K, Pathak OK, Alurkar VM. Acute coronary syndrome in elderly-the difference compared with young in intensive care unit of a tertiary hospital in western Nepal. Journal of Clinical and Diagnostic Research. 2009; 3:1289-96. doi: 10.4103/1319-3767.54746

[6] Beyraghi N, Tonekaboni SH, Vakili G. [Anxiety and depression in patients admitted in cardiac care unit, Taleghani Hospital, Tehran, Iran, 2003 (Persian)]. Hormozgan Medical Journal. 2006; 9(4):261-64.

[7] Sharif F, Shoul AR, Jannati M, Zare N, Kojouri J. [Effect of cardiac rehabilitation on anxiety and depression in CABG patients (Persian)]. Journal of Rehabilitation. 2011;12(2):8-13.

[8] Taghipour B, Sharif Nia HS, Soleimani MA, Hekmat Afshar M, Shahidi Far SS. Comparison of the clinical symptoms of myocardial infarction in the middle-aged and elderly. Journal of Kermanshah University of Medical Sciences. 2014; 18(5):281-89.

[9] Mesbah M. [Islamic approach to spiritual health (Persian)]. $2^{\text {nd }}$ ed. Tehran: Hoghooghi Publication; 2013.

[10] Momennasab M, Moattari M, Abbaszade A, Shamshiri B. [Spiritual experience of heart attack patients: A qualitative study (Persian)]. Journal of Qualitative Research in Health Sciences. 2013; 1(4):284-97.

[11] Omidvari S. [Spiritual health, its nature and the instruments used (Persian)]. Iranian Journal of Psychiatry and Clinical Psychology. 2010; 16(3):274-279.

[12] Abbasi M, Azizi F, Shamsi Kooshki E, Naseri Rad M, Akbari Lake M. [Conceptual and operational definitions of mental health: a methodological study (Persian)]. Medical Ethics. 2012; 6(20):11-44.

[13] Gaur KL, Sharma M. Measuring Spiritual Health: Spiritual Health Assessment Scale (SHAS). International Journal of Innovative Research and Development. 2014; 3(3):63-7.

[14] McCoubrie RC, Davies AN. Is there a correlation between spirituality and anxiety and depression in patients with advanced cancer? Supportive Care in Cancer. 2006; 14(4):379-85. doi: $10.1007 /$ s00520-005-0892-6
آرامش را ايجاد مي كند [Tس]]. در مطالعاتى نيز ارتباط مثبت بين

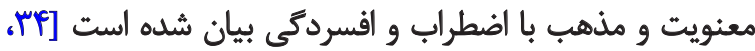

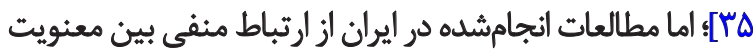

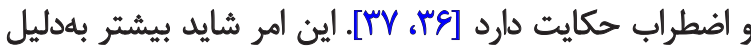

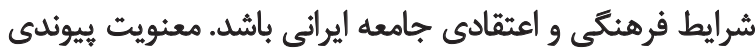

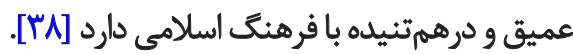
نتايج اين مطالعه از وجود ارتباط معنادار بين سلامت معنوى

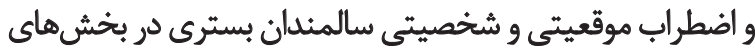

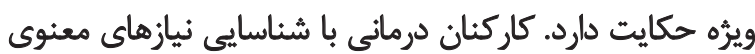

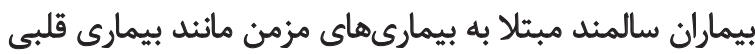

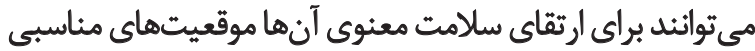

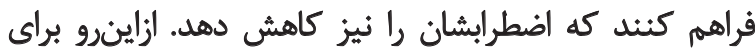

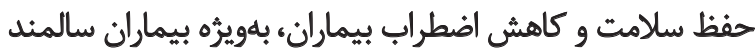

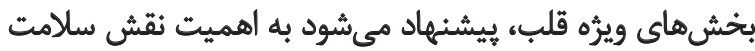

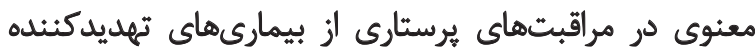

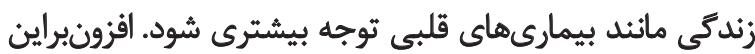

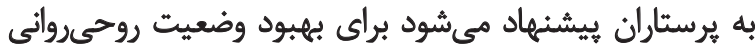

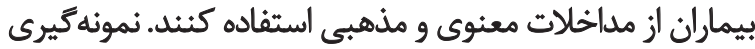

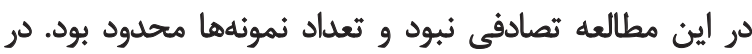

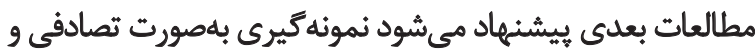
در جمعيت آمارى بيشترى بيشياد في شود شود.

$$
\text { تشكر وقدردانى }
$$

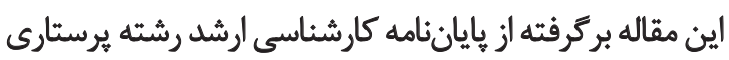

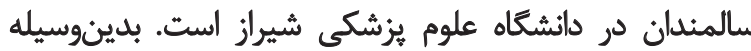

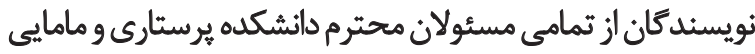

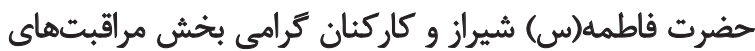

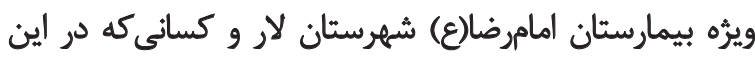
تحقيق شركت كردهاند، تشكر و قدردانى مى كنيند. 
[15] Papazisis G, Nicolaou P, Tsiga E, Christoforou T, SapountziKrepia D. Religious and spiritual beliefs, self-esteem, anxiety, and depression among nursing students. Nursing \& Health Sciences. 2014; 16(2):232-38. doi: 10.1111/nhs.12093

[16] Moghimian M, Salmani F. [The study of correlation between spiritual well-being and hope in cancer patients referring to Seyyedo Shohada Training-Therapy Center of Isfahan University of Medical Sciences, 2010 (Persian)]. Qom University of Medical Sciences Journal. 2012; 6(3):40-45.

[17] Unterrainer HF, Lewis A, Fink A. Religious/spiritual wellbeing, personality and mental health: a review of results and conceptual issues. Journal of Religion \& Health. 2014; 53(2):382-92. doi: $10.1007 /$ s10943-012-9642-5

[18] Ardelt M, Landes SD, Gerlach KR, Fox LP. Rediscovering internal strengths of the aged: The beneficial impact of wisdom, mastery, purpose in life, and spirituality on aging well. In: Sinnott JD, editor. Positive Psychology: Advances in Understanding Adult Motivation. California: Springer; 2013, p. 98-119. doi: 10.1007/978-1-4614-7282-7_7

[19] Farahani M, Farzad V, Mokhtari F. [Spielberger state-trait anger expression inventory examine the psychometric properties (Persian)]. Journal of Psychology and Educational Sciences. 2008; 38(4):165-88.

[20] Jahani A, Rejeh N, Heravi-Karimooi M, Hadavi A, Zayeri F, Khatooni A. The relationship between spiritual health and quality of life in patients with coronary artery disease. Islamic Lifestyle Centered on Health. 2013; 1(2):17-21. doi: 10.5812/ilch.8575

[21] Sang OC, Won-Hee L. Relationship between spiritual health and depression of patients with hematological malignancies. Paper Presented at: The $32^{\text {nd }}$ Annual Oncology Nursing Society Congress; 2011 Oct 27; Las Vegas, Nevada, USA.

[22] Habibi A, Savadpoor M. [Spiritual well-being in cancer patients who undergo chemotherapy (Persian)]. Journal of Health and Care. 2011; 13(3):16-20.

[23] Assarroudi A, Jalilvand M, Oudi D, Akaberi A. [The relationship between spiritual well-being and life satisfaction in the nursing staff of Mashhad Hasheminezhad Hospital (2011) (Persian)]. Modern Care. 2012; 9(2):156-62.

[24] Rowe MM, Allen RG. Spirituality as a means of coping with chronic illness. American Journal of Health Studies. 2004; 19(1):62-67.

[25] Alahbakhshian M, Jafarpour Alavi M, Parvizi S, Haghani H. A Survey on relationship between spiritual wellbeing and quality of life in multiple sclerosis patients. Zahedan Journal of Research in Medical Sciences. 2010; 12(3):29-33.

[26] Hashemzadeh A, Garooci Farshi M, Halabianloo G, Maleki Rad A. [The study of effectiveness of relaxation and distraction techniques training in anxiety reduction in cardiac patients (Persian)]. Arak Medical University Journal. 2011; 14(56):97-105.

[27] Tahmasbi H, Akbarzade H, Darvishi H. [Trait anxiety and state anxiety before coronary angiography (Persian)]. Journal of Family Health. 2012; 1(1):41-46.

[28] Breslau J, Miller E, Jin R, Sampson NA, Alonso J, Andrade LH, et al. A multinational study of mental disorders, marriage, and divorce. Acta Psychiatrica Scandinavica. 2011; 124(6):474-86. doi 10.1111/j.1600-0447.2011.01712.x
[29] Meyer D, Paul R. A cross-national examination of marriage and early life stressors as correlates of depression, anxiety, and stress. Family Journal. 2011;19(3):274-80. doi 10.1177/1066480711406678

[30] Lee KE, Lee YE. The relationship between the spiritual health, anxiety and pain in hospitalized cancer patients. Korean Journal of Hospice \& Palliative Care. 2015; 18(1):25-34. doi 10.14475/ kjhpc.2015.18.1.25

[31] Golkar MK, Sadat Banijamali S, Bahrami H, Hatami HR, Ahadi H. Effect of spiritual therapy on blood pressure, anxiety and quality of life in patients with high blood pressure. Journal of Kermanshah University of Medical Sciences. 2014; 18(8):462-70.

[32] Saidimehr S, Gravandi S, Ezadmehr A, Mohammadi MJ, Hasani M. [Study of relationship between spiritual health and depression among elderly people (Persian)]. Journal of Ilam University of Medical Sciences. 2015; 23(3):16-25.

[33] Mohammadi F, Babaee M. [Effects of participation in support groups on Alzheimer's family caregivers' strain and spiritual wellbeing (Persian)]. Iranian Journal of Ageing. 2011; 6(1):29-37.

[34] Pargament KI, Koenig HG, Tarakeshwar N, Hahn J. Religious struggle as a predictor of mortality among medically ill elderly patients: a 2-year longitudinal study. Archives of Internal Medicine. 2001; 161(15):1881-885. doi: 10.1001/archinte.161.15.1881

[35] Koenig HG, Larson D, McCullough M. Handbook of religion and health. Oxford: Oxford University Press; 2000.

[36] Mahboobi M, Eetemadi M, Khorasani E, Ghiasi A. [The relationship between spiritual health and social anxiety disorder in veterans (Persian)]. Iranian Journal of Military Medicine. 2012; 14(3):186-91.

[37] Bolhari J, Naziri G, Zamanian S. [Spiritual efficacy of therapeutic approaches to reduce depression anxiety and stress in women with breast cancer (Persian)]. Journal of Sociology and Women. 2012; 3(1):85-115.

[38] Abbasi M, Shamsi Kooshki E, Abolghasemi M. [An Introduction to spiritual health (Persian)]. Tehran: Hoghooghi Publication; 2013. 